\title{
Modern Technique for Real Photon Radiative Correction Calculations
}

\author{
I. A. Shershan* and T. V. Shishkina ${ }^{\dagger}$ \\ Department of Theoretical Physics and Astrophysics, \\ Belarusian State University, 4 Nezavisimosti Ave., 220030 Minsk, Belarus
}

(Received 23 August, 2020)

\begin{abstract}
The problem of the bremsstrahlung contribution calculation as a part of the radiative corrections in the case of single gauge boson production was discussed. It was shown that the hard photon bremsstrahlung contribution can be divided into the finite and divergent terms. The exact calculation of soft photon bremsstrahlung and infrared part of hard photon bremsstrahlung was presented in frame of the dimensional regularization scheme. Numerical analysis of radiative corrections to the cross sections of single gauge boson production was performed.
\end{abstract}

PACS numbers: 12.15.Lk, 12.60.Cn, 14.60.Cd

Keywords: photon, electron, gauge boson, electroweak interaction, radiative corrections, linear colliders

DOI: https://doi.org/10.33581/1561-4085-2021-24-2-184-191

\section{Introduction}

The calculation of radiative corrections to the cross sections of the processes of elementary particles interactions is an important and timeconsuming problem of quantum field theory. The presence of unphysical infrared (IR) and ultraviolet (UV) divergences requires the usage of specific methods for their parameterization and cancelation. In general, the existence of divergences imposes significant restrictions on the possibility of direct numerical analysis of the observed quantities, including the total and differential cross sections of the processes under consideration. In this regard, the analytical covariant description of the processes is highly essential.

Conventionally, the contribution of the lowest-order radiative corrections can be divided into two categories: the contribution of virtual particles, or one-loop corrections (V-terms) and the contribution of real photons, or bremsstrahlung (R-terms). UV-divergences

\footnotetext{
*E-mail: shershan@bsu.by

${ }^{\dagger}$ E-mail: shishkina.tatiana.v@gmail.com
}

are eliminated by taking into account the contribution of counterterms in accordance with the chosen renormalization scheme. IRdivergences are eliminated by taking into account the R-contribution.

The problems associated with the Vcontribution are substantially resolved. Taking into account the contribution of real photons is a much more complicated task, requiring an exceptional approach to each individual process and its kinematics. Due to this, the problem of accounting for the $\mathrm{R}$-contribution requires a larger number of unique analytical results. It should be noted that Belarusian scientists also played an important role in developing effective methods for covariant analytical calculations of the $\mathrm{R}$-contribution for various processes [1-3].

In this paper, results concerning a number of problems that arise in calculating the Rcontribution for various processes were obtained. In accordance with the common methods, the contribution of soft photons is calculated using the dimensional regularization method, as well as calculation of the contribution of hard bremsstrahlung with the separation of the IR diverging part for processes based on electron- 
photon collisions, which will take place at the International Linear Collider (ILC) $[4,5]$ and are important for the study of the Standard Model variations [6-11].

\section{The soft photon bremsstrahlung}

The simplest way to taking into account the bremsstrahlung contribution for elimination of IR divergences is the soft-photon approximation. In case if the final photons have sufficiently low energy, in the propagators of the processes amplitude 4-momentum of photon can be neglected and the squared matrix element for the bremsstrahlung process can be represented as follows:

$$
d \sigma_{\mathrm{B}}=\delta_{\mathrm{R}}^{\mathrm{SB}} \cdot d \sigma_{\mathrm{B}}
$$

The factor $\delta_{\mathrm{R}}^{\mathrm{SB}}$ can be written as

$$
\delta_{\mathrm{R}}^{\mathrm{SB}}=-\frac{\alpha}{2 \pi^{2}}\left(\sum_{i}^{m} I_{i}+\sum_{i>j}^{m} A_{i j}^{C} A_{i j}^{I F} I_{i j}\right)
$$

where $A_{i j}^{C}, A_{i j}^{I F}$ is the charge asymmetry and state asymmetry coefficients, respectively. They are equal to +1 , if particles with momenta $p_{i}$ and $p_{j}$ have the same charges / both are finite (initial) particles, and -1 otherwise. Summation is performed over the momenta of external charged particles. follows:

The functions $I_{i}$ and $I_{i j}$ are expressed as

$$
\begin{array}{r}
I_{i}=1 / m_{i}^{2} I\left(p_{i}\right), \\
I_{i j}=1 / x_{i j} I\left(p_{i}, p_{j}\right),
\end{array}
$$

where $x_{i j}=2 p_{i} p_{j}, p_{i}^{2}=m_{i}^{2}$ and functions $I\left(p_{i}\right)$ and $I\left(p_{i}, p_{j}\right)$ are the following integrals

$$
\begin{gathered}
I\left(p_{i}, p_{j}\right)=\int_{0}^{\Delta E} \frac{d^{3} q}{(2 \pi)^{3} 2 q^{0}} \frac{1}{\left(p_{i} q\right)\left(p_{j} q\right)} \\
I\left(p_{i}\right)=I\left(p_{i}, p_{i}\right)
\end{gathered}
$$

with integration over the bremmstrahlung photon 4-momentum $q$.

To calculate these integrals, one make the following changes of variables

$$
p=\zeta p_{i}, \quad k=p_{j} .
$$

Here $\zeta$ can be obtained from equation $(p-k)^{2}=0$, and the 4 -momentum $p-k$ is isotropic. In this case, the initial integral can be rewritten in the form:

$$
I\left(p_{i}, p_{j}\right)=\zeta \int_{0}^{\Delta E} \frac{d^{3} q}{(2 \pi)^{3} 2 q^{0}} \frac{1}{(p q)(k q)} .
$$

Performing analytical continuation to the $n$ dimensions and transforming integration to integration over angles, one can obtain [12]

$$
I\left(p_{i}, p_{j}\right)=\frac{2}{(2 \sqrt{\pi})^{n} \Gamma(n / 2-1)} \int_{0}^{1} d x \frac{1}{\mu^{n-4}} \int_{0}^{\Delta E}\left(q^{0}\right)^{n-5} d q^{0} \int_{0}^{\pi}(\sin \theta)^{n-3} d \theta \frac{1}{\left[\left(u^{0}\right)^{2}-|\vec{u}|^{2} \cos \theta_{x}\right]^{2}}
$$

Here $x$ is Feynman parameter and $u=p x+k(1-$ $x)$. Integrating over the photon energy, one can come to the following expression:

$$
\begin{aligned}
& \frac{1}{\mu^{n-4}} \int_{0}^{\Delta E} d q^{0}\left(q^{0}\right)^{n-5}=\frac{(\Delta E / \mu)^{n-4}}{n-4} \\
& =\frac{1}{n-4}\left[1+(n-4) \ln \frac{\Delta E}{\mu}+\ldots\right] .
\end{aligned}
$$


The soft photon emission is isotropic. Therefore, it is always possible to do such choice a coordinate frame, that one of the axes coincides in direction with one of the vectors, and accordingly, $\theta_{\alpha}=\theta$ :

$$
\int_{0}^{\pi} \frac{(\sin \theta)^{n-3} d \theta}{\left[\left(u^{0}\right)^{2}-|\vec{u}|^{2} \cos \theta\right]^{2}}=\int_{-1}^{1} \frac{\left(\left(1-\xi^{2}\right)^{n / 2-2} d \xi\right.}{\left[\left(u^{0}\right)^{2}-|\vec{u}|^{2} \xi\right]^{2}}=\int_{-1}^{1} \frac{d \xi}{\left[\left(u^{0}\right)^{2}-|\vec{u}|^{2} \xi\right]^{2}}\left[1+\frac{1}{2}(n-4) \ln \left(1-\xi^{2}\right)+\ldots\right]
$$

Comparing the last two expressions and discarding all terms except linear on $1 /(n-4)$ we obtain

$$
\begin{array}{r}
\frac{1}{\mu^{n-4}} \int_{0}^{\Delta E} d q^{0}\left(q^{0}\right)^{n-5} \int_{0}^{\pi} \frac{(\sin \theta)^{n-3} d \theta}{\left[\left(u^{0}\right)^{2}-|\vec{u}|^{2} \cos \theta\right]^{2}}=\int_{-1}^{1} \frac{d \xi}{\left[\left(u^{0}\right)^{2}-|\vec{u}|^{2} \xi\right]^{2}}\left[\frac{1}{n-4}+\ln \frac{\Delta E}{\mu}+\frac{1}{2} \ln \left(1-\xi^{2}\right)\right] \\
=2\left[\frac{1}{n-4}+\ln \frac{2 \Delta E}{\mu}\right] / u^{2}+\frac{u^{0}}{|\vec{u}| \cdot u^{2}} \ln \frac{u^{0}-|\vec{u}|}{u^{0}+|\vec{u}|} .
\end{array}
$$

Expanding $n$ near 4 gives

$$
\begin{array}{r}
I\left(p_{i}, p_{j}\right)=\zeta \frac{1}{2(2 \pi)^{2}}\left(R_{1}+R_{2}\right), \\
R_{1}=\int_{0}^{1} \frac{d x}{u^{2}}\left[-\Delta^{\mathbf{I R}}+\ln \frac{4 \Delta E^{2}}{\mu^{2}}\right], \\
R_{2}=\int_{0}^{1} \frac{d x}{u^{2}} \frac{u^{0}}{|\vec{u}|} \ln \frac{u^{0}-|\vec{u}|}{u^{0}+|\vec{u}|} .
\end{array}
$$

Here $\Delta^{\mathbf{I R}} \equiv \frac{1}{\bar{\epsilon}}=\frac{2}{4-n}-\gamma_{E}+\ln 4 \pi$ is IR-regulator in the $\overline{\mathrm{MS}}$-scheme. Using the obvious expression

$$
\begin{array}{r}
u^{2}=k^{2}+2 k \cdot(p-k)=k^{2}+2 v l, \\
l=p^{0}-k^{0}, \quad v=\frac{2 k \cdot(p-k)}{l}=\frac{p^{2}-k^{2}}{2 l},
\end{array}
$$

it is easy to obtain an expression for $R_{1}$ :

$$
\begin{gathered}
R_{1}=\int_{0}^{1} \frac{d x}{k^{2}+2 v l}\left[-\Delta^{\mathbf{I R}}+\ln \frac{4 \Delta E^{2}}{\mu^{2}}\right] \\
=\frac{1}{p^{2}-k^{2}} \ln \frac{p^{2}}{k^{2}}\left[-\Delta^{\mathbf{I R}}+\ln \frac{4 \Delta E^{2}}{\mu^{2}}\right] .
\end{gathered}
$$

The integral (13) is identical to the integral calculated by 't Hooft and Veltman [13] and is equal to

$$
\begin{array}{r}
R_{2}=\frac{2}{p^{2}-k^{2}}\left[\frac{1}{4} \ln ^{2} \frac{u^{0}-|\vec{u}|}{u^{0}+|\vec{u}|}+\operatorname{Li}_{2}\left(1+\frac{u^{0}+|\vec{u}|}{v}\right)\right. \\
\left.+\operatorname{Li}_{2}\left(1+\frac{u^{0}-|\vec{u}|}{v}\right)\right]\left.\right|_{u=k} ^{u=p}
\end{array}
$$

Thus, the functions $I_{i} I_{i j}$ will be expressed as follows:

$$
I_{i}=\pi\left[-\Delta^{\mathbf{I R}}+\ln \frac{4 \Delta E^{2}}{\mu^{2}}+\frac{p^{0}}{|\mathbf{p}|} \ln \frac{p^{0}-|\mathbf{p}|}{p^{0}+|\mathbf{p}|}\right]
$$




$$
\begin{array}{r}
I_{i j}=2 \pi \frac{\zeta\left(x_{i j}\right)}{\zeta^{2} m_{i}^{2}-m_{j}^{2}}\left[\frac{1}{2} \ln \frac{\zeta^{2} m_{i}^{2}}{m_{j}^{2}}\left(-\Delta^{\mathbf{I R}}+\ln \frac{4 \Delta E^{2}}{\mu^{2}}\right)\right. \\
\left.+\left[\frac{1}{4} \ln ^{2} \frac{u^{0}-|\mathbf{u}|}{u^{0}+|\mathbf{u}|}+\operatorname{Li}_{2}\left(1-\frac{u^{0}+|\mathbf{u}|}{v}\right)+\left.\operatorname{Li}_{2}\left(1-\frac{u^{0}-|\mathbf{u}|}{v}\right)\right|_{u=p_{j}} ^{u=\zeta p_{i}}\right]\right] .
\end{array}
$$

In equations $(17,18)$ the expressions

$$
\begin{gathered}
\zeta=\frac{x_{i j}+\sqrt{x_{i j}^{2}-4 m_{i}^{2} m_{j}^{2}}}{2 m_{i}^{2}}, \\
d v=\frac{\zeta^{2} m_{i}^{2}-m_{j}^{2}}{2\left(\zeta p_{i}^{0}-p_{j}^{0}\right)}, \\
x_{i j}=2 p_{i} \cdot p_{j}
\end{gathered}
$$

are used.

\section{The hard bremsstrahlung}

The soft photon bremsstrahlung contribution depends on the resolution of collider energy. However in the soft photon approximation this energy should be much smaller than the interaction energy (including the masses of particles). To avoid this dependence one can take into account the hard photon bremsstrahlung contribution. Since the kinematics of the bremsstrahlung process is different from the kinematics of the initial process, it is impossible to fully algorithmize the calculation of the contribution of the bremsstrahlung. It must be noted that the tensor structure of the matrix elements of the bremsstrahlung processes allows naturally to separate finite and the IR-divergent parts of the squared matrix elements of the processes under consideration:

$$
\left|\mathcal{M}_{\mathrm{R}}\right|^{2}=\left|\mathcal{M}_{\mathrm{R}}^{\mathrm{F}}\right|^{2}+\left|\mathcal{M}_{\mathrm{R}}^{\mathrm{IR}}\right|^{2},
$$

and IR-divergent term can be factorized with matrix element in Born approximation $\left|\mathcal{M}_{\mathrm{R}}^{\mathrm{IR}}\right|^{2} \propto$ $\mid \mathcal{M}_{\mathrm{B}}$. Respectively, the differential cross section of bremsstrahlung process can be also factorized:

$$
d \sigma_{\mathrm{R}}=\delta_{\mathrm{R}}^{\mathrm{IR}} \cdot d \sigma_{\mathrm{B}}
$$

Let us consider the special case of the single gauge boson production process with the additional bremsstrahlung photon:

$$
\begin{aligned}
& e^{-}\left(p, m_{e}\right)+\gamma(k, 0) \rightarrow \\
& \rightarrow C^{-}\left(p_{1}, m_{c}\right)+N^{0}\left(k_{1}, m_{n}\right)+\gamma(q, 0) .
\end{aligned}
$$

To describe the kinematics of the initial process (without a finite photon), two parameters are needed. Often Mandelstam invariants are used in covariant calculations:

$$
s=(p+k)^{2}, \quad t_{1} \equiv-Q^{2}=\left(k-k_{1}\right)^{2} .
$$

For describing kinematics of bremsstrahlung process it is necessary three additional invariants:

$$
\begin{gathered}
s_{1}=\left(p_{1}+q\right)^{2} ; \\
s_{2}=\left(p_{1}+k_{1}\right)^{2} ; \\
t_{2}=(p-q)^{2} .
\end{gathered}
$$

The expression of the total cross section for the such type of processes has the following form:

$$
\sigma_{\mathrm{R}}=\frac{(2 \pi)^{-4}}{4\left(s-m_{e}^{2}\right)^{2}} \int\left|\mathcal{M}_{\mathrm{R}}\right|^{2} \frac{d t_{1} d s_{1} d s_{2} d t_{2}}{8 \sqrt{-\Delta_{4}}}
$$

Comparison with the eq. (21) gets $\delta_{\mathrm{R}}^{\mathrm{IR}}$ for the following expression and can be written as follows: 


$$
\begin{aligned}
\delta_{\mathrm{R}}^{\mathrm{IR}} & =-\frac{\alpha}{\pi^{2}} \int\left(\frac{m_{e}^{2}}{\left(m_{e}^{2}-t_{2}\right)^{2}}+\frac{m_{c}^{2}}{\left(s_{1}-m_{c}^{2}\right)^{2}}-\frac{Q^{2}+m_{e}^{2}+m_{c}^{2}}{\left(m_{e}^{2}-t_{2}\right)\left(s_{1}-m_{c}^{2}\right)}\right) \frac{d s_{1} d t_{2} d s_{2}}{\sqrt{-\Delta_{4}}} \\
& =-\frac{\alpha}{\pi^{2}}\left(m_{e}^{2} \delta_{1}+m_{1}^{2} \delta_{2}+\left(m_{1}^{2}+m_{e}^{2}-t_{1}\right) \delta_{3}\right) .
\end{aligned}
$$

This equation is depend on all new invariants. Here $\Delta_{4}$ is the Gram determinant. All kinematic boundaries can be obtained from the condition $\Delta_{4}=0$. Gram determinant can be presented in the form $\Delta_{4}=-\lambda\left(s_{2}^{+}-s_{2}\right)\left(s_{2}^{-}-s_{2}\right), \lambda \equiv$ $\lambda\left(s_{1}, t_{1}, m_{e}^{2}\right)$. It is easy to verify that the factor $\delta_{\mathrm{R}}^{\mathrm{IR}}$ coincides with $\delta_{\mathrm{R}}^{\mathrm{SB}}$, but is expressed using invariants of different kinematics.

For another invariants one has the following kinematical restrictions for IR-parametrization in the laboratory system with $\vec{p}=0$ :

$$
\begin{array}{r}
t_{2}^{ \pm}=-s_{1}+t_{1}+\frac{1}{2 s_{1}}\left[\left(s_{1}+m_{1}^{2}\right)\left(s_{1}+m_{1}^{2}-t_{1}\right)\right. \\
\left. \pm \sqrt{\lambda\left(s_{1}, m_{1}^{2}, t_{1}\right) \lambda\left(s_{1}, m_{1}^{2}, 0\right)}\right], \\
\bar{s}_{1}=\frac{\left(s+t_{1}-m_{e}^{2}-M^{2}\right)\left(m_{e}^{2} M^{2}-s t_{1}^{2}\right)}{\left(s-m_{e}^{2}\right)\left(M^{2}-t_{1}\right)}, \\
\underline{s}_{1}=m_{1}^{2}+F \\
t_{1}=M_{1}^{2}-\frac{1}{2 s}\left[\left(s-m_{e}^{2}\right)\left(s-m_{1}^{2}+M_{1}^{2}\right)\right. \\
\left.\mp\left(s-m_{e}^{2}\right) \sqrt{\lambda\left(s, m_{1}^{2}, M_{1}^{2}\right)}\right] .
\end{array}
$$

Here $F=2 m_{1} \Delta E$. Integration over $s_{2}$ is trivial. Let us calculate several integrals by $t_{2}$ :

$$
\begin{aligned}
\int_{t_{2}^{-}}^{t_{2}^{+}} \frac{d t_{2}}{\left(t_{2}-m_{e}^{2}\right)^{2}} & =\frac{\sqrt{\lambda}}{m_{e}^{2}\left(s_{1}-m_{1}^{2}\right)}, \\
\int_{t_{2}^{-}}^{t_{2}^{+}} d t_{2} & =\sqrt{\lambda} \frac{\left(s_{1}-m_{1}^{2}\right)}{s_{1}}, \\
\int_{t_{2}^{-}}^{t_{2}^{+}} \frac{d t_{2}}{t_{2}-m_{e}^{2}} & =\ln \left[\frac{1-\beta}{1+\beta}\right],
\end{aligned}
$$

where $\beta=\sqrt{\lambda} /\left(s_{1}+m_{e}^{2}-t_{1}\right)$. Using these results and additionally preforming integration over $s_{2}$ one can give following results for $\delta_{1}$ and $\delta_{2}$ in the limit $\Delta E \rightarrow 0$ :

$$
\begin{gathered}
\delta_{1}=-\frac{1}{m_{e}^{2}} \ln \left[\frac{2 \Delta E m_{1}}{\bar{s}_{1}-m_{1}^{2}}\right], \\
\delta_{2}=-\frac{1}{m_{1}^{2}}\left\{\ln \left[\frac{2 \Delta E m_{1}}{\bar{s}_{1}-m_{1}^{2}}\right]+\ln \left[\frac{\bar{s}_{1}}{m_{1}^{2}}\right]\right\} .
\end{gathered}
$$

The last form factor $\delta_{3}$ has the form

$$
\delta_{3}=\int_{m_{1}^{2}+F}^{\bar{s}_{1}} \frac{\ln \left[\frac{1-\beta}{1+\beta}\right]}{\sqrt{\lambda}\left(s_{1}-m_{1}^{2}\right)} d s_{1} .
$$

This integral is not calculated by conventional methods. Let us calculate it approximately. For this purpose, one must separate the finite and divergent parts of $\delta_{3}$ :

$$
\delta_{3}=\delta_{3}^{\mathrm{IR}}+\delta_{3}^{\mathrm{F}} .
$$

The divergent part must be determined as accurately as possible. Therefore, we use the expansion of the logarithm

$$
\ln \left[\frac{1-\beta}{1+\beta}\right]=\sum_{i=0}^{\infty} \frac{\beta^{2 i+1}}{2 i+1}
$$

and integrate it. Assembling the parts containing the divergences, it is easy to see that this is the expansion of the following function:

$$
\delta_{3}^{\mathrm{IR}}=\frac{1}{\sqrt{\lambda_{t}}} \ln \left[x_{t}\right] \ln \left[\frac{2 \Delta E m_{1}}{\bar{s}_{1}-m_{1}^{2}}\right] .
$$

Here the notations

$$
\begin{gathered}
\lambda_{t}=\lambda\left(t_{1}, m_{e}^{2}, m_{1}^{2}\right), \\
\beta_{t}=\sqrt{\lambda_{t}} /\left(m_{e}^{2}+m_{1}^{2}-t_{1}\right), \\
x_{t}=\frac{1-\beta_{t}}{1+\beta_{t}}
\end{gathered}
$$


are introduced.

In the ultrarelativistic approximation $\left(m_{e} \rightarrow\right.$

The final expression for $\delta_{\mathrm{R}}^{\mathrm{IR}}[14]$ is $0)$

$$
\delta_{3}=\int_{m_{1}^{2}+F}^{\bar{s}_{1}} \frac{\ln \left[\frac{s_{1}}{m_{e}^{2}}\right]-\frac{1}{2} \ln \left[\frac{s_{1}-t_{1}}{s}\right]}{\left(s_{1}-t_{1}\right)\left(s_{1}-m_{1}^{2}\right)} d s_{1} .
$$

$$
\begin{aligned}
\delta_{\mathrm{R}}^{\mathrm{IR}}= & -\frac{\alpha}{2 \pi}\left[\ln \frac{4 \Delta E^{2} m_{c}^{2}}{\left(\bar{s}_{1}-m_{c}^{2}\right)^{2}}\left[2-\frac{1}{\beta_{t}} \ln x_{t}\right]+\Re\left\{\ln s_{1}-2 \ln \frac{m_{c}^{2}-t}{s_{1}-t}\right.\right. \\
& -\ln ^{2}\left(s_{1}-t\right)+\ln \left(\bar{s}_{1}-m_{c}^{2}\right)\left(2 \ln \left(s_{1}-t\right)-\ln m_{e}^{2} s_{1}\right) \\
& \left.\left.+\ln m_{e}^{2} s_{1} \ln \frac{m_{c}^{2}\left(s_{1}-t\right)}{-t}-\operatorname{Li}_{2} \frac{s_{1}}{m_{c}^{2}}+\operatorname{Li}_{2} \frac{s_{1}}{t}+\left.2 \operatorname{Li}_{2} \frac{s_{1}-t}{m_{c}^{2}-t}\right|_{s_{1}=m_{c}^{2}} ^{s_{1}=\bar{s}_{1}}\right\}\right] .
\end{aligned}
$$

It can be obtained as a result of integration and comparison of the form factors expression.

\section{Numerical analysis}

In order to evaluate the contribution of the finite part of the R-contribution, let us conduct a numerical analysis for a number of processes, taking into account the above calculations. Comparing the obtained results with the results of other authors calculation, one can approximately estimate the contribution of the finite part of the R-contribution and make conclusions about the need to take it into account. Differential radiative corrections for various processes of single gauge bosons production in high-energy electron-photon collisions are shown in Fig. 1. It shows that the absolute value of the radiative corrections increases with increasing energy, also the $W$-boson production process demonstrates the highest value of corrections, which reach $-35 \%$ for energy of colliding beams $\sqrt{s}=1 \mathrm{TeV}$ and momentum transfer $|Q|=170 \mathrm{GeV}$.

As it was noted above, a numerical analysis can be successfully performed only at the level of the total cross sections of processes. For numerical calculations the adaptive quasi Monte-Carlo method Vegas was chosen, and the cutting angle was taken equal to $\Delta \vartheta=20^{\circ}$. The total relative radiative corrections for the same processes are presented in Fig. 2. At the interaction energy $\sqrt{s}=1 \mathrm{TeV}$ the radiative corrections for the processes of neutral gauge bosons production amount about $5 \%$ in absolute value, which, in general, is consists with the data of other researchers. However for the $W$-boson production process at the same energy, the value of radiative corrections reaches $30 \%$. Obviously an additional peak should be observed in the finite R-contribution, which will lead to the increase of the relative radiative correction of the process to $20 \%$ value.

\section{Conclusion}

In elementary particle physics calculation of the lowest-order radiative corrections is crucial for increasing the accuracy analysis of the characteristics of processes. The usage of covariant methods in calculations of radiative corrections is extremely important both for confirming the predictions of the Standard Model and for the searching physics beyond it. Dimensional regularization method for parametrization of unphysical divergences is important to consistently parametrization both 

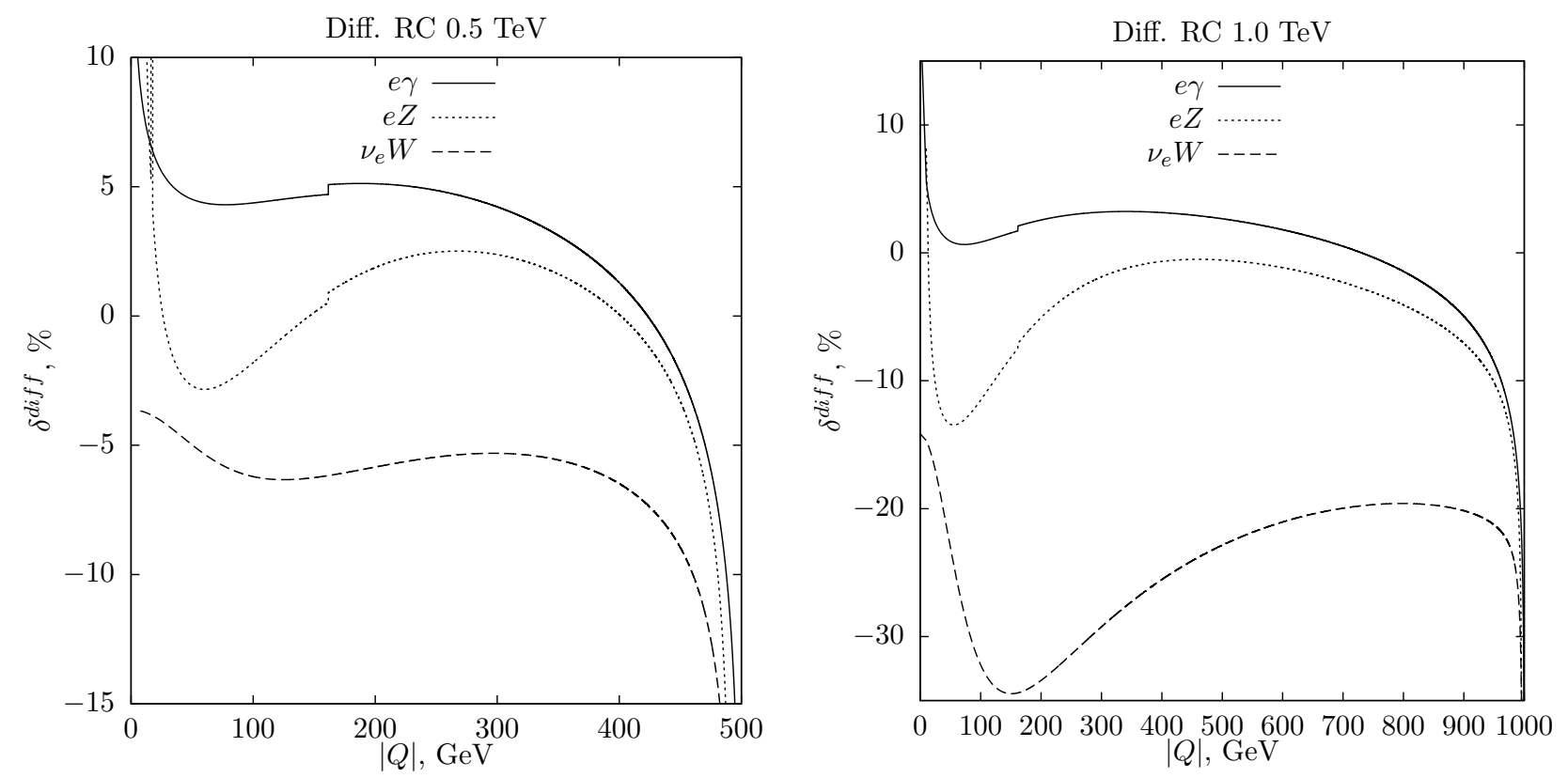

FIG. 1: The radiative corrections to differential cross section for a set of processes.

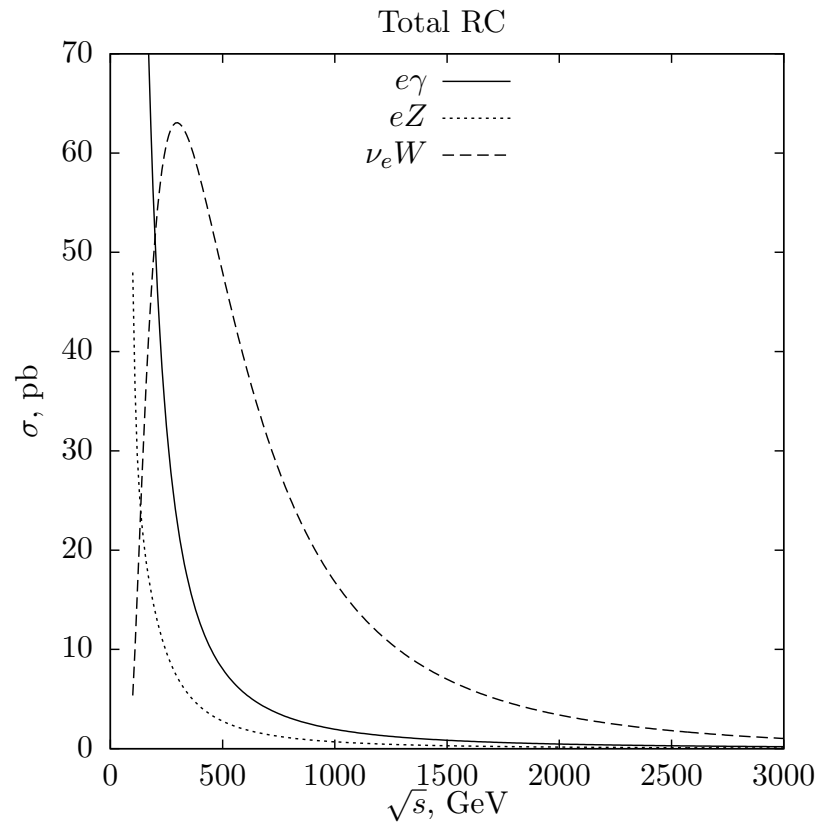

a)

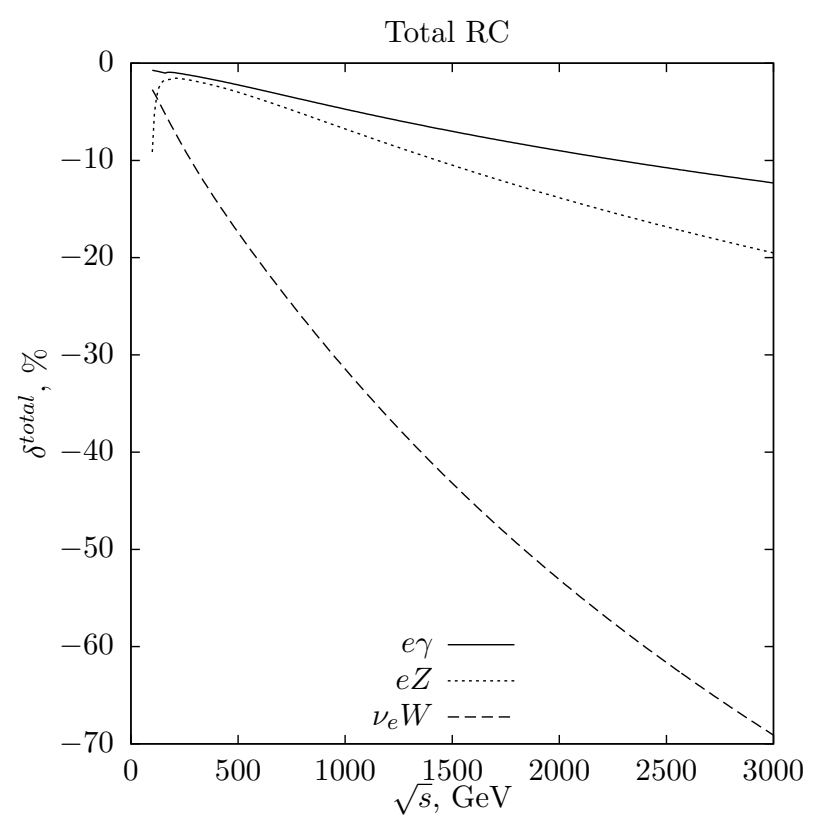

b)

FIG. 2. The total cross sections including the lowest order radiative corrections a) and relative radiative corrections b) for a set of processes.

IR and UV singularities.

The obtained in this paper results can be applied in calculations of the R-contribution as a part of radiative corrections for processes in experiments at all types colliders of elementary particles. The used dimensional regularization method allows account for the bremsstrahlung in the most correct and modern way. Analytical 
accounting of the IR-divergent part of the contribution of hard bremsstrahlung is important for the numerical stability of the calculations of the R-contribution. It allows to obtain the results in Lorentz-invariant form, as well as eliminate the dependence on the experimental parameters. The ultra-relativistic approximation is good at experimental energies about $100 \mathrm{MeV}$, therefore the obtained data are sufficiently accurate. Numerical analysis showed that it required to take into account the finite part of the R-contribution. In particular, for the processes of the $e \gamma \rightarrow$ $C N(\gamma)$ type, contribution to the relative radiative corrections is exceptionally positive, and at high interaction energies it can reach $10-20$ percents.

\section{References}

[1] D. Y. Bardin and N. Shumeiko, Nuclear Physics B 127, 242 (1977).

[2] T. Kuchto and N. Shumeiko, Nuclear Physics B 219, 412 (1983).

[3] T. Kukhto, N. Shumeiko, and S. Timoshin, Journal of Physics G: Nuclear Physics 13, 725 (1987).

[4] T. Behnke, J. E. Brau, B. Foster, J. Fuster, M. Harrison, J. M. Paterson, M. Peskin, M. Stanitzki, N. Walker, and H. Yamamoto, arXiv preprint arXiv:1306.6327 (2013).

[5] I. Ginzburg, G. Kotkin, S. Panfil, V. Serbo, and V. I. Telnov, Nuclear Instruments and Methods in Physics Research 219, 5 (1984).

[6] H. Aihara, T. Barklow, U. Baur, J. Busenitz, S. Errede, T. Fuess, T. Han, D. London, J. Ohnemus, R. Szalapski, et al., in Electroweak symmetry breaking and new physics at the TeV scale (World Scientific, 1996), pp. 488-547.
[7] T. Barklow, U. Baur, F. Cuypers, S. Dawson, D. Errede, S. Errede, S. Godfrey, T. Han, P. Kalyniak, K. Riles, et al., arXiv preprint hep$\mathrm{ph} / 9611454$ (1996).

[8] A. Denner and S. Dittmaier, Nuclear Physics B 540, 58 (1999).

[9] A. Denner and S. Dittmaier, Nuclear physics B 398, 265 (1993).

[10] M. Böhm and S. Dittmaier, Nuclear Physics B 409, 3 (1993).

[11] T. Shishkina, Nonliner phenomena in complex systems 11, 60 (2011).

[12] A. Akhundov, D. Bardin, L. Kalinovskaya, and T. Riemnann, Fortschritte der Physik 44, 373 (1996).

[13] G. t Hooft and M. Veltman, Nuclear Physics B 153, 365 (1979).

[14] D. Y. Bardin, Nucl. Phys. 127, 242 (1977). 